\title{
Assessing the physical healthcare gap among patients with severe mental illness: large real-world investigation from Italy
}

\author{
Giovanni Corrao, Matteo Monzio Compagnoni, Valeria Valsassina and Antonio Lora
}

\section{Background}

One critical barrier to the uptake of mental health programmes is the so-called physical healthcare gap, a concern raised by the unattended physical comorbidity and early mortality of persons with severe mental illness.

\section{Aims \\ To evaluate the extension of physical healthcare gap among persons with severe mental illness under chronic drug therapies.}

\section{Method}

A population-based cohort study was carried out, using Lombardy healthcare utilisation databases. Prevalent patients treated with blood pressure-, lipid- or glucose-lowering agents were identified in January 2017. Among these, those who were receiving care for depression, schizophrenia, bipolar disorder or personality disorder formed the study cohort. A reference cohort was randomly selected from prevalent patients treated with chronic therapies without signs of severe mental disorders, to be matched with study cohort members for gender, age and number of previous contacts with the National Health System. One-year adherence to healthcare was measured through the proportion of days covered (drug adherence), and exposure to selected recommendations (clinical control adherence).

\section{Results}

The 55162 patients with severe mental illness were less likely to have high adherence to blood pressure-lowering, lipid-lowering or antidiabetic agents than the reference cohort by $-24 \%(95 \% \mathrm{Cl}$ -26 to $-22 \%),-10 \%(95 \% \mathrm{Cl}-14$ to $-6 \%)$ and $-25 \%(95 \% \mathrm{Cl}-29$ to $-21 \%)$, respectively. The 9250 patients with diabetes and severe mental illness had $-18 \%(95 \% \mathrm{Cl}-22 \%$ to $-13 \%)$ reduced likelihood to meet recommendations for the clinical management of diabetes, compared with the reference cohort.

\section{Conclusions}

Adherence to chronic drug therapies was sensibly worse among patients living with mental illness than those without signs of mental disorders.

\section{Keywords}

Mental healthcare; physical healthcare gap; drug adherence; severe mental illness; healthcare utilisation database.

\section{Copyright and usage}

(c) The Author(s), 2021. Published by Cambridge University Press on behalf of the Royal College of Psychiatrists. This is an Open Access article, distributed under the terms of the creative Commons Attribution licence (http://creativecommons.org/ licenses/by/4.0/), which permits unrestricted re-use, distribution, and reproduction in any medium, provided the original work is properly cited.
Mental health epidemiologists and scholars of mental healthcare services are moving beyond traditional measures of incidence and prevalence to include treatment gap $^{1,2}$ and assessment of unmet needs in psychiatry. ${ }^{3,4}$ A main issue in this field includes the physical healthcare gap, a concern raised by the relatively frequent but highly unattended physical comorbidity ${ }^{5}$ and early mortality of persons with severe mental illness. ${ }^{6,7}$

Patients with chronic physical conditions may be non-adherent to recommended care for several reasons, including their disbelief in the efficacy of treatment, ${ }^{8}$ the presence of barriers such as adverse effects, ${ }^{9,10}$ and lack of help and support from family members ${ }^{11}$ or health professionals. In addition, it is noteworthy that nonsomatic diseases, like mental health disorders, might also affect patients' ability or willingness to adhere to recommended treatments. Even when people receive care from mental health services, mental health professionals may not give adequate attention to the physical assessment of patients treated for psychiatric disorders. ${ }^{12}$ Indeed, having both a physical and mental health condition still results in more complicated treatments and poorer outcomes than having either problem alone. ${ }^{13}$

Assessing the extent to which non-adherence to recommended treatments for chronic somatic diseases might be a potentially avoidable concomitant effect of a non-somatic treatable condition (i.e. mental disorders) may be an important first step in improving patient adherence, the therapeutic alliance between physicians and patients, the outcomes of medical treatment ${ }^{14,15}$ and ultimately, to provide further evidence to support the need for integrating physical and mental health in public health policies.

\section{Aims of the study}

The aim of the present study was to assess the extension of physical healthcare gap among persons with severe mental disorders under chronic drug therapy, evaluating the association between exposure to mental disorders and adherence to recommended healthcare for chronic somatic diseases. We identified three large, regional population-based cohorts of patients who were under chronic pharmacological therapy with blood pressure-lowering, lipid-lowering or glucose-lowering agents, some of whom had severe mental disorders, to investigate whether and to what extent mental illness affected the short-term adherence to recommended healthcare.

\section{Method}

\section{Data sources}

The study was based on the computerised healthcare utilisation (HCU) databases of Lombardy, an Italian northern region accounting for almost 10 million inhabitants (about 16\% of the national population). In Italy, all citizens have equal access to healthcare provided by the National Health Service (NHS). Its management in Lombardy is associated with an automated system of HCU 
databases, which include a variety of information on the beneficiaries of the regional health service (virtually all residents in the region), such as diagnosis at discharge from public or private hospitals, out-patient drug prescriptions, specialist visits and diagnostic examinations provided fully or partly free of charge, by the NHS. In addition, a specific automated system concerning mental healthcare gathers data from regional Departments of Mental Health (DMHs) accredited by the NHS (i.e. the so-called 'Italian Mental Health Information System'). This system provides demographic information and diagnostic and therapeutic codes for patients receiving specialist mental healthcare by the regional $\mathrm{DMH}$ facilities. These various types of data can be interconnected through a record-linkage procedure, since a unique individual identification code is used among all databases for each NHS beneficiary, to trace the complete healthcare pathway of each resident. To preserve privacy, each identification code is automatically anonymised, which can only be reversed by the regional authority upon request from judicial authorities. Further details on HCU database use in the field of mental healthcare have been reported elsewhere. ${ }^{12,16}$ Diagnostic and drug therapy codes used for drawing records and fields from the considered databases are reported in Supplementary Table 1 available at https://doi.org/10.1192/bjo. 2021.998.

\section{Selecting the study and reference cohorts}

Beneficiaries of the NHS who, on 1 January 2017 (index date), were aged 18 years or older, were resident in Lombardy for at least 2 years and were receiving treatment with blood pressure-lowering, lipidlowering or antidiabetic agents, were identified and included as three separated groups. An individual was considered to be receiving treatment with a given drug therapy if, during the 2-year period before the index date (i.e. 2015-2016), they had received at least three consecutive dispensations of that drug therapy. Drug dispensations were considered consecutive (uninterrupted) if the timespan between the coverage end of one prescription and the beginning of the following prescription was 60 days or shorter, being the defined daily dose metrics assumed for calculating drug coverage. It is noteworthy that the three groups of prevalent patients treated with blood pressure-lowering, lipid-lowering or antidiabetic agents were not independent, since a patient on therapy with two (or three) of the considered medications was included in the corresponding two (or three) groups. Patients who died or moved to another region or country within 1 year of the index date were excluded from the groups (i.e. at least 1 year of observation was required for every participant).

Within each of the considered groups, patients diagnosed with a severe mental disorder who were receiving care from a mental health service at the index date were identified and considered as belonging to the study cohort. Patients were defined as receiving care when they had an active record in the Mental Health Information System of having received a diagnosis of depression, schizophrenia, bipolar disorder or personality disorder at any time before the index date, and were still receiving care for the disorder at the index date.

A reference cohort suitable to be used as comparator for the study cohort was generated. Patients who were eligible to be selected as comparators were those who belonged to the groups being treated with a given drug therapy (the same groups that generated the study cohorts), but had no mental health service provision recorded in the Mental Health Information System. For each study cohort member, up to three eligible comparators were randomly selected to be matched for gender, age at index date ( \pm 1 year) and number of contacts with the NHS (i.e. drug dispensations, hospital admissions, out-patient visits and procedures) in the 2 years before the index date.
Both study and reference cohort members were followed from the index date until 1 year after the index date (end-point of follow-up).

\section{Assessing adherence to healthcare}

Starting from the index date, all medications dispensed during the following year to patients belonging to study and reference cohorts were recorded. The duration of each prescription was calculated by dividing the total amount of the drug prescribed by the defined daily dose. Adherence to drug therapy was measured by the ratio between the cumulative number of days in which the drug was available and days of the overall follow-up (proportion of days covered; PDC) ${ }^{17,18}$ Adherence was categorised according with PDC value as very low (PDC $\leq 25 \%)$, low (26-50\%), intermediate $(51-74 \%)$ and high $(\geq 75 \%)$.

Because we have no information about in-hospital dispensed drugs, therapeutic regimens observed before hospital admission was assumed as continuously administered during the hospital stay, taking into account the so-called 'immeasurable time bias'. ${ }^{19}$

Out-patient clinical controls, including assessments of lipid profile (total and HDL cholesterol and triglycerides), serum creatinine, glycated haemoglobin, urine albumin excretion and dilated eye examinations dispensed to cohort members on drug therapy with antidiabetic agents, were identified. A patient was considered as adherent to these recommendations when they had at least two glycated haemoglobin assays and at least one of the other evaluations, annually. ${ }^{20,21}$ Overall, a high adherence to recommended clinical controls was considered to be reached for those patients who underwent all, or almost all, recommendations (i.e. when at least four of the five controls were performed during the first year after the index date). ${ }^{22}$

\section{Additional measurements}

Baseline characteristics of study and reference cohort members included comorbidities and cotreatments (antithrombotic, antiarrhythmics and antineoplastic agents, digitalis, nitrates, nonsteroidal anti-inflammatory drugs (NSAIDs) and drugs for pulmonary diseases). Comorbidities was identified from in-patient diagnoses and out-patient drug treatments experienced within the 2 years before the index date. In addition, patients were categorised according to the Multisource Comorbidity Score (MCS), a new index of patients' clinical status derived from in-patient diagnostic information and out-patient drug prescriptions, provided by the regional Italian data and validated for outcome prediction. ${ }^{23}$

\section{Data analysis}

Baseline characteristics of study and reference cohort members were compared by means of absolute standardised differences (we considered an absolute standardised difference of $<0.10$ as negligible ${ }^{24}$ ). Comparisons regarding adherence to healthcare recommendations were made by means of chi-square test, or its version for the trend, where proper $(P<0.05$ was considered to be significant).

Multivariable conditional logistic regression was fitted for modelling the odds ratio and $95 \%$ confidence interval, for the association between the exposure and the outcome(s) of interest. Cardinal exposure was the condition of having a diagnosis of mental disorder or not, i.e. to belong to the study or reference cohort, respectively. A patient was considered as having experienced the outcome when, during the follow-up period, at least $75 \%$ of the drug was available (i.e. the patient had a high drug adherence, $\mathrm{PDC} \geq 75 \%$ ) or at least four of the five controls were performed (i.e. high clinical control adherence). Adjustments for the covariates listed in the above 'Additional measurements' section were performed. Stratified 


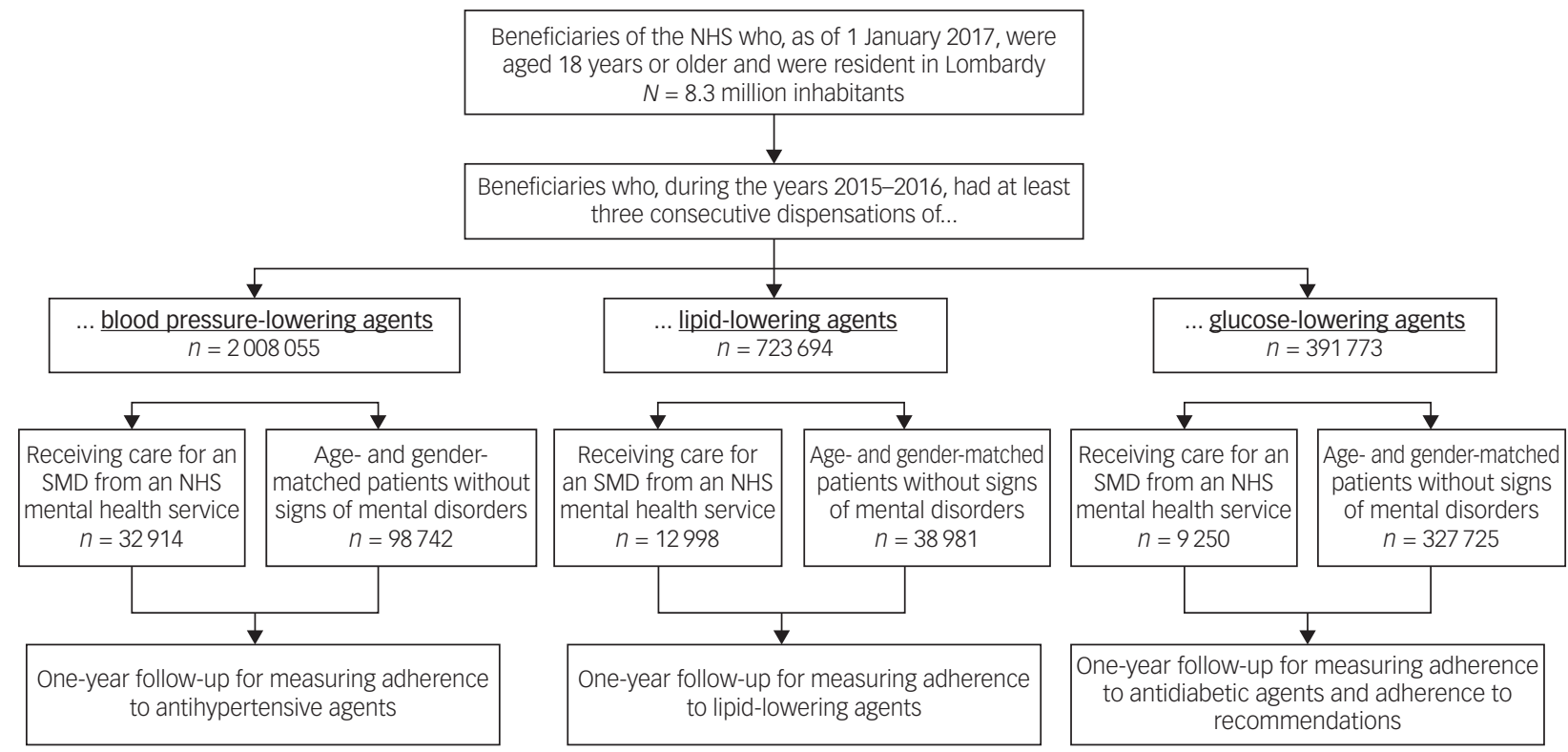

Fig. 1 Flow chart showing criteria for eligibility of prevalent patients treated with blood pressure-, lipid-and glucose-lowering agents and, within each category of drug therapy, patients with and without mental disorders. NHS, National Health Service; SMD, severe mental disorders (depression, schizophrenia, bipolar disorder and personality disorders).

analyses were performed according to predefined stratification variables (diagnostic categories of mental disorders, gender, age classes and categories of MCS). In other terms, adjusted conditional logistic regression was fitted as described, separately for each strata of the stratification variables. Between-strata homogeneity of odds ratios (type of mental disorder and gender) and along-strata trend (categories of age and MCS) were tested for model estimates. The hypothesis of homogeneity among strata was tested with the chisquare test, whereas trends in odds ratio were tested with the statistical significance of the regression coefficient of the recoded variable, obtained by scoring the corresponding categories of adherence. The regression coefficients of the outcome risk trends between strata were compared with the $z$-test.

The exposure $\rightarrow$ outcome association was expressed by (odds ratio-1) $\times 100$, a percentage variation of the likelihood of high adherence to recommendations among patients with a severe mental disorder versus patients without a severe mental disorder. Statistical evidence of reduced adherence was assumed when a negative value of the quantity (lower bound of the confidence interval at $95 \%$ of the (odds ratio-1) $\times 100$ ) was obtained. Conversely, significant increased adherence was assumed when a positive value of the quantity (upper bound of the confidence interval at $95 \%$ of the (odds ratio -1$) \times 100$ ) was obtained.

To verify the robustness of our findings, we adopted different ways for the categorisation of drug and controls adherence, i.e. a patient was considered having experienced the outcome when at least 70 or $80 \%$ of the follow-up period was covered by the drug therapy (instead of the $75 \%$ cut-off adopted for the main analysis), or at least three of the five controls were performed (instead of the four controls adopted in the main analysis).

The software SAS (version 9.4 for Windows; SAS Institute, North Carolina, USA) was used to perform all analyses.

\section{Ethical issues}

The Ethical Committee of the University of Milano-Bicocca evaluated the protocol (protocol number 497, year 2019) and established that the study was exempt from informed consent (according to General Authorization for the Processing of Personal Data for
Scientific Research Purposes issued by the Italian Privacy Authority on 10 August 2018; https://www.gpdp.it/web/guest/ home/docweb/-/docweb-display/docweb/9124510); provides sufficient guarantees of individual records anonymity and was designed according to quality standards of good practice of observational research based on secondary data.

\section{Results}

\section{Patients}

The process of cohort selection is shown in Fig. 1. Among the 8.3 million inhabitants from Lombardy aged 18 years or older on 1 January 2017, 2008055 (24\%), 723694 (9\%) and 391773 (5\%) were on treatment with blood pressure-, lipid- and glucose-lowering agents, respectively. Around $2 \%$ were receiving care at the facilities of regional DMHs, forming the study cohorts of 32 914, 12998 and 9250 prevalent patients treated with blood pressure-, lipid- and glucose-lowering agents with a diagnosis of a severe mental disorder. Patients without a severe mental disorder were 1:3 matched, forming the reference cohort.

The baseline characteristics of study and reference cohort members are compared in Table 1. The mean age (s.d.) was around 65 (s.d. 12) years, and women were systematically more represented. Although co-medications were essentially similar in study and reference cohort members (with a few exceptions; for example, NSAIDs were more frequently prescribed among study cohort members than comparators), patients with mental disorders had a worse clinical profile on average than reference cohort members.

\section{Adherence to healthcare}

As shown in Fig. 2, during the first year of follow-up, cohort members with mental disorders experienced lower adherence to the corresponding specific drug therapy compared with their comparators. With the exception of serum creatinine and dilated eye examination, patients with comorbid diabetes and a severe mental disorder had lower adherence to individual recommendations for 


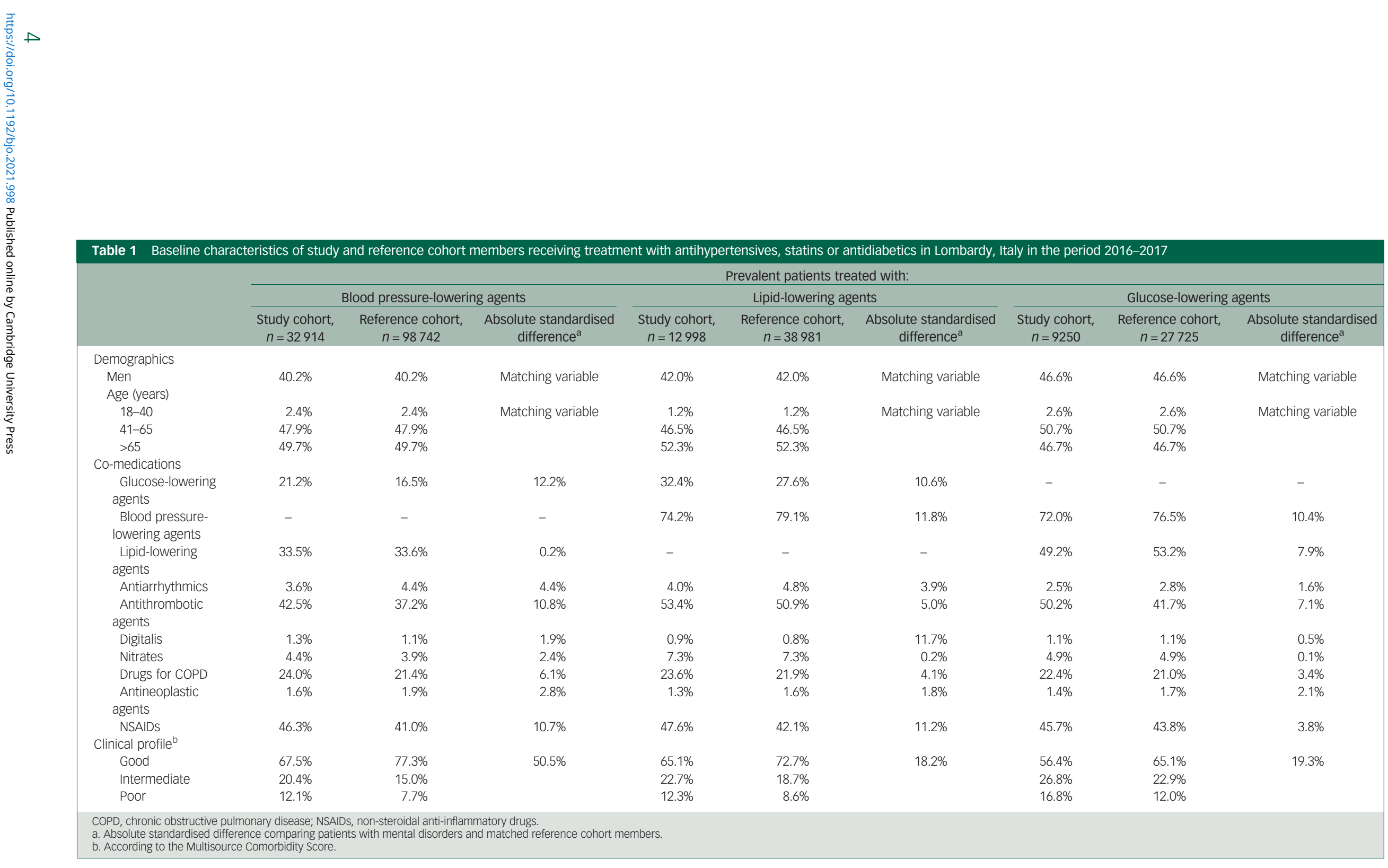


Drug adherence / blood pressure-lowering agents

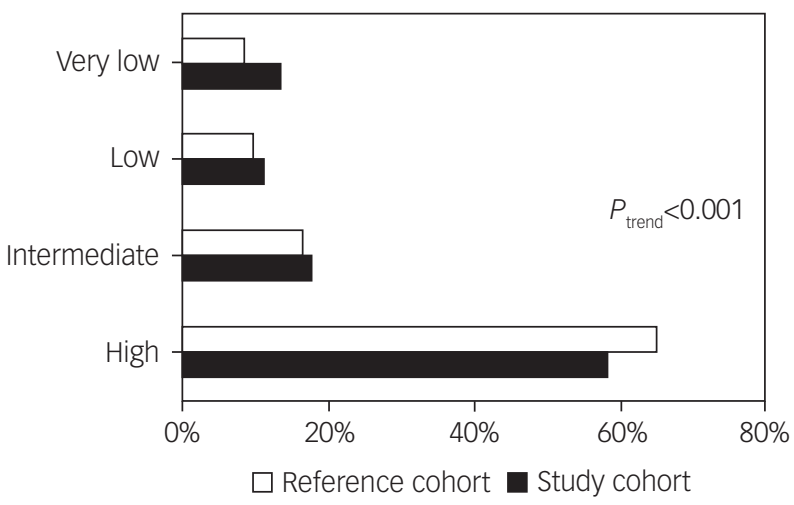

Glucose-lowering agents / drug adherence

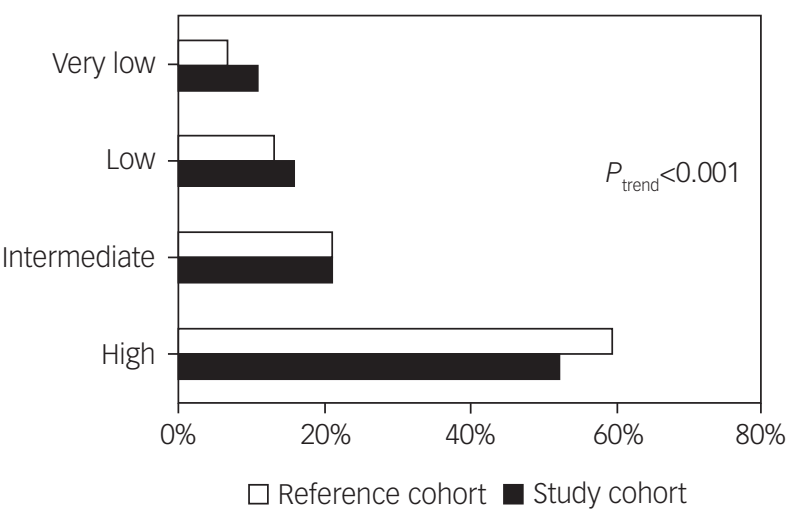

Lipid-lowering agents / drug adherence

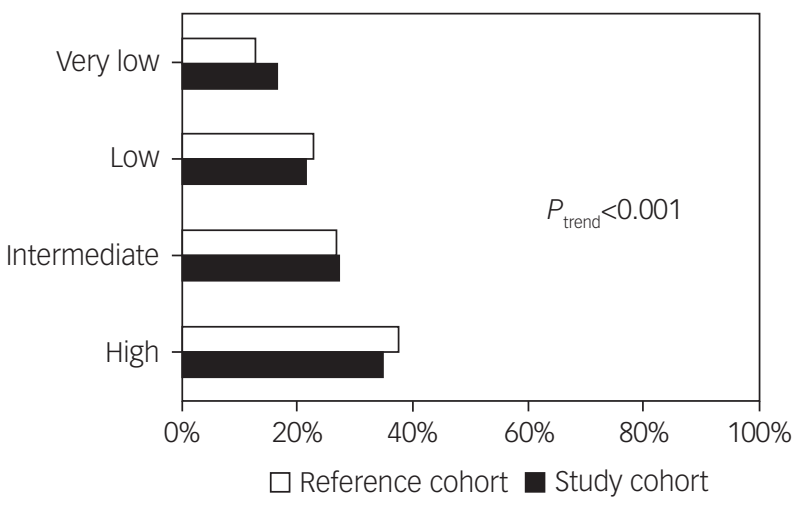

Glucose-lowering agents / controls adherence

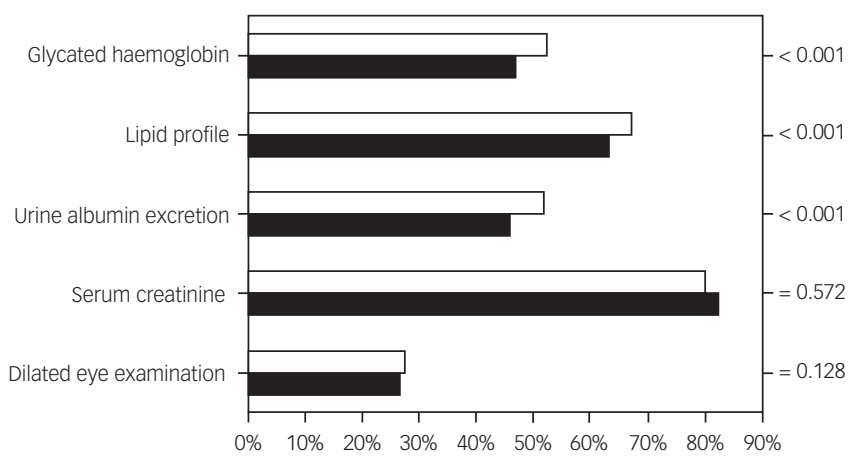

$\square$ Reference cohort $\square$ Study cohort

Fig. 2 Distribution of reference and study cohort members according to the categories of adherence to drug therapy and clinical controls.

Adherence to each pharmacological therapy is categorised as very low $(\leq 25 \%)$, low $(26-50 \%)$, intermediate $(51-74 \%)$ and high $(\geq 75 \%)$ proportion of days covered by drug prescriptions.

clinical controls than the reference cohort (mainly glycated haemoglobin and urine albumin excretion). Study cohort members consistently had significant lower odds of high adherence to drugs and clinical controls than comparators, and the gap between study and reference cohort members was higher for glucose- and blood pressure-lowering agents than for lipid-lowering agents (Fig. 3). Indeed, compared with the reference cohort, patients with a severe mental disorder had reduced likelihood of high adherence to blood pressure-lowering, lipid-lowering or antidiabetic drug therapies of $-24 \%$ ( $95 \%$ CI -26 to $-22 \%$ ), $-10 \%$ (95\% CI -14 to $-6 \%)$ and $-25 \%$ (95\% CI -29 to $-21 \%)$, respectively. A reduced likelihood of high adherence to recommendations for diabetes management of $-18 \%$ (95\% CI -22 to $-13 \%)$ was also observed among patients with diabetes and severe mental disorder, compared with those with diabetes but no mental disorder.

Stratified analysis showed that type of mental disorder, gender and clinical profile were significant effect modifiers (Table 2). That is, the gap between patients with and without mental disorders was even more pronounced than that obtained from the comparison of whole cohorts, among men (always), among patients affected by personality disorders (usually, except for the adherence to lipid-lowering agents) and among those with good clinical profile (restricted to patients with diabetes). There was no evidence that the adherence gap associated with mental disorders differed among age categories.

The relationship between the presence of a severe mental disorder and high adherence to the recommended healthcare did not substantially change when varying the thresholds for the categorisation of high adherence levels (Supplementary Table 2).

\section{Discussion}

In our study, the 55162 patients with severe mental illness who were receiving chronic drug therapies had a likelihood of high adherence to blood pressure-lowering, lipid-lowering or antidiabetic agents of $-24 \%,-10 \%$ and $-25 \%$, respectively, compared with patients without severe mental illness who were receiving the same drugs. Consistently, the 9250 patients with comorbid diabetes and a severe mental disorder had $18 \%$ lower likelihood of meeting recommendations for the management of diabetes compared with those with diabetes without a severe mental disorder. The healthcare gap of patients with mental disorders was wider for men and those with personality disorders (although it also affected patients with schizophrenia, bipolar disorder and, to a lesser extent, depression), and had major extension among patients with good clinical profile. These findings should be interpreted in light of the design from which they were generated. Because patients with severe mental disorders were receiving treatment from mental health services, we expect that they had an advantage in terms of medical assistance and healthcare access, which patients without mental disorders did not. This means that the real gap between patients with and without mental disorders is expected to be wider than that observed in our study for individuals with severe mental disorder who do not receive treatment from mental health services.

Our findings are consistent with several meta-analysis and primary studies emphasising treatment gap and unmet needs in psychiatry. ${ }^{25}$ First, acceptability of medications by people living 
Risk reduction (and 95\% confidence interval) of being properly treated with recommended healthcare

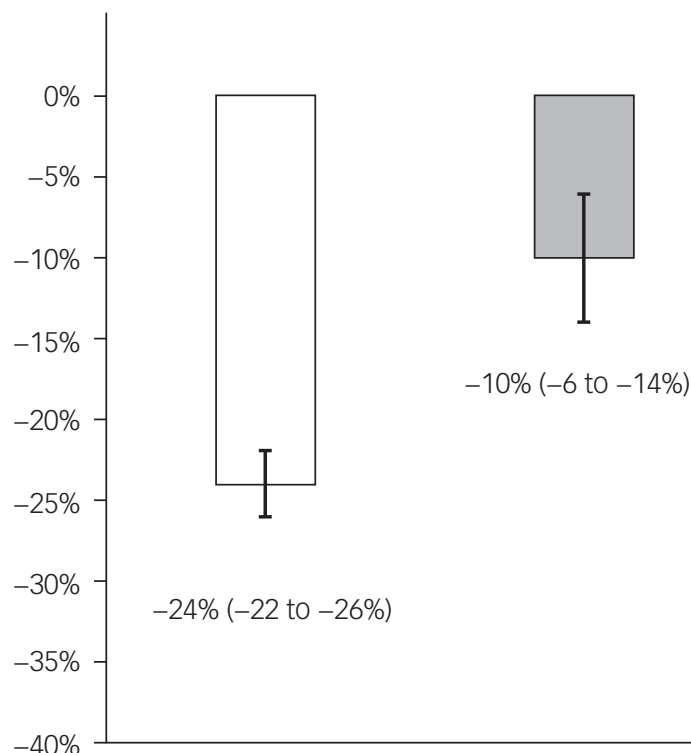

Adherence to blood pressure-lowering drugs

Adherence to glucose-lowering drugs
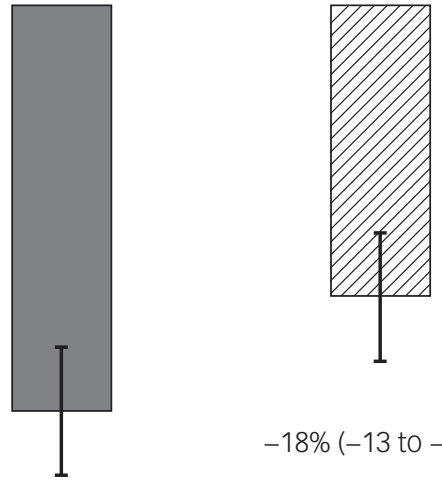

$-18 \%(-13$ to $-22 \%)$

$-25 \%(-21$ to $-29 \%)$

Adherence to lipid-lowering drugs

Adherence to clinical controls for diabetes management

Fig. 3 Percentage variation of the likelihood of high adherence to recommendations among patients with severe mental disorder compared with those without evidence of severe mental disorder, and corresponding $95 \%$ confidence intervals. Percentage variation of the likelihood of high adherence to recommendations was derived from the quantity (odds ratio-1) $\times 100$. The corresponding $95 \%$ confidence interval was obtained from the $95 \%$ confidence interval of the odds ratio. The latter was estimated with conditional logistic regression. Estimates are adjusted for the covariates listed in

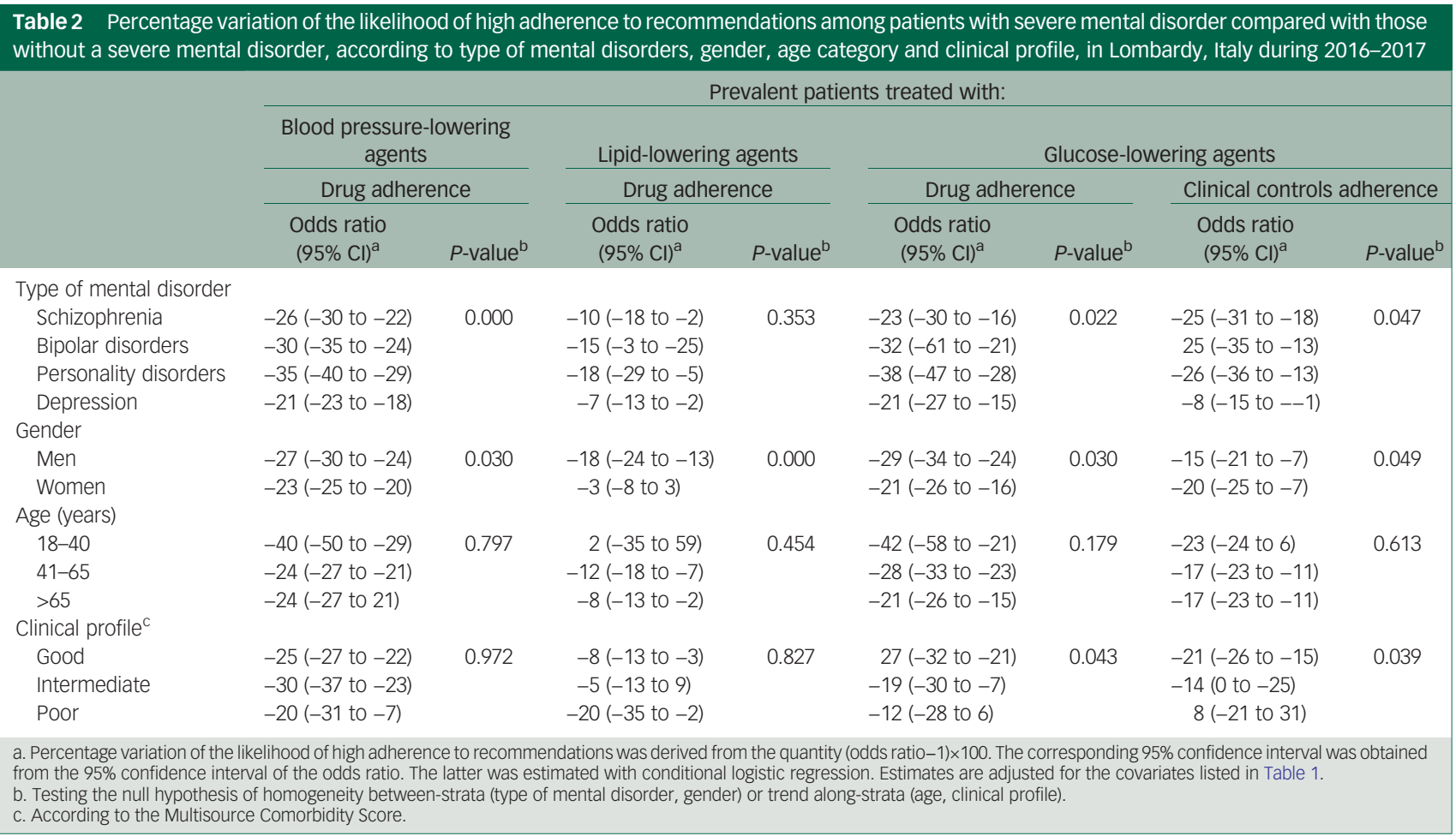


with severe and persistent mental illness has been described as problematic, and adherence to medications is poor. ${ }^{26}$ Second, these issues are often overlooked and/or dismissed by healthcare professionals and policy makers. ${ }^{27}$ For example, physical health concerns of people on antipsychotics were not addressed by prescribing psychiatrists in a UK study. ${ }^{28}$ Finally, the lifespan of persons with serious mental illness has been consistently reported as shortened compared with those without serious mental illness. ${ }^{29-34}$ Up to $50 \%$ of their mortality excess is considered potentially preventable through providing timely and high-quality healthcare. ${ }^{35-40}$ Our findings allow for speculation that more careful control of adherence to drug treatments and medical controls, mainly for diabetes and hypertension, would reduce the gap between patients with and without severe mental illness, and perhaps reduce the increased risk of cardiovascular morbidity and mortality among patients with mental disorders. ${ }^{6}$ This implies that the management of the physical health of patients with severe mental disorders needs to be urgently addressed by physicians and decision makers.

The present study is unique in several respects. The investigation is based on routine clinical practice delivered by the Italian National Health System, a free-of-charge universal healthcare system covering essential health needs of all citizens. High-quality interconnectable individual data on out-patient and in-patient services supplied by the NHS, including healthcare provided by the public DMHs, offers the unique opportunity of tracing the complete care pathway of large, unselected populations. The resulting realworld evidence are free from selective participation or recall bias.

Our study has several limitations. Common sources of exposure misclassification include treatments dispensed by private services, as well as out-of-pocket payments. A pitfall of the present study concerns lack of clinical data (e.g. severity of hypertension, hyperlipidaemia and diabetes, other related complications, and comorbidities) as well as socioeconomic information (e.g. economic status and family guardianship), potentially affecting the adherence to chronic drug therapies. Although socioeconomic status can be confidently ruled out because we have previously found that in Lombardy income and educational differences play no role in the persistence on antihypertensive drug treatment, ${ }^{40}$ and despite the fact that the effect of some clinical factors has been carefully controlled in our study by means of stratified analysis, a deeper knowledge of clinical and social traits would have allowed us to more clearly explain some of the findings.

In conclusion, one of the critical barriers to the uptake of mental health programmes is the so-called physical healthcare gap, a concern raised by the relatively frequent but highly unattended physical comorbidity and early mortality of persons with severe mental illness. Our paper showed that patients living with mental illness who needed chronic drug therapy with blood pressure-lowering, lipid-lowering or antidiabetic agents were treated worse than patients without signs of mental disorders but with the same need of chronic drug therapies. The gap in sufficient utilisation of adequate treatment options was particularly pronounced for patients with personality disorders (although it also affected patients with schizophrenia, bipolar disorder and, to a lesser extent, depression), of male gender and with a good general clinical profile.

We hope that the current paper may motivate the research in this field and that further investigations address the causes of non-adherence and effective interventions to overcome the physical healthcare gap of patients with severe mental illness. Meanwhile, individual (from patients, families, psychiatrists, physicians and decision makers) and system-level actions, promoting a joint approach to physical and mental health by mental health professionals and general practitioners, should be considered a priority to adequately address an often ignored problem with a major impact on public health.
Giovanni Corrao PhD National Centre for Healthcare Research and

Pharmacoepidemiology, University of Milano-Bicocca, Italy; and Unit of Biostatistics, Epidemiology and Public Health, Department of Statistics and Quantitative Methods, University of Milano-Bicocca, Italy; Matteo Monzio Compagnoni $\mathbb{D}$, PhD, National Centre for Healthcare Research and Pharmacoepidemiology, University of MilanoBicocca, Italy; and Unit of Biostatistics, Epidemiology and Public Health, Department of Statistics and Quantitative Methods, University of Milano-Bicocca, Italy;

Valeria Valsassina, BSC, National Centre for Healthcare Research and

Pharmacoepidemiology, University of Milano-Bicocca, Italy; and Unit of Biostatistics, Epidemiology and Public Health, Department of Statistics and Quantitative Methods, University of Milano-Bicocca, Italy; Antonio Lora, MD, National Centre for Healthcare Research and Pharmacoepidemiology, University of Milano-Bicocca, Italy; and Department of Mental Health and Addiction Services, Azienda Socio Sanitaria Territoriale di Lecco, Italy

Correspondence: Matteo Monzio Compagnoni. Email: matteo.monziocompagnoni@ unimib.it

First received 22 Feb 2021, final revision 2 Aug 2021, accepted 12 Aug 2021

\section{Supplementary material}

Supplementary material is available online at https://doi.org/10.1192/bjo.2021.998

\section{Data availability}

The data that support the findings of this study are available from the Lombardy Region, but restrictions apply to the availability of these data, which were used under license for the current study and so are not publicly available. Data are, however, available from the authors upon reasonable request, and with permission of Lombardy Region.

\section{Author contributions}

G.C. and A.L. contributed to the initial study idea, interpretation of the results and drafting of the manuscript. M.M.C. and V.V. contributed to the preparation of the data-set for the analysis and to the data analysis. G.C., A.L. and M.M.C. contributed to the protocol and interpretation of the results. A.L. contributed to the interpretation of pharmacological and clinical prospective results, and reviewed the manuscript. M.M.C. also contributed to the drafting of the manuscript. All of the authors contributed to the critical revision of the manuscript. G.C. is the guarantor of this work and, as such, had full access to all of the data in the study and takes responsibility for the integrity of the data and the accuracy of the data analysis.

\section{Funding}

This study was supported and funded by the Italian Ministry of Education, University and Research ('Fondo d'Ateneo per la Ricerca' portion, year 2018; grant number: 2018-ATE0485). The funding source had no role in the design of the study; the collection, analysis and interpretation of the data; the writing of the manuscript or the decision to approve publication of the finished manuscript.

\section{Declaration of interest}

G.C. received research support from the European Community, the Italian Medicines Agency and the Italian Ministry of Education, University and Research. He took part in a variety of projects that were funded by pharmaceutical companies (i.e. Novartis, GlaxoSmithKline, Roche, Amgen and Bristol Myers Squibb). He also received honoraria as a member of the advisory board for Roche. All remaining authors declare no other potential conflicts of interest relevant to this article.

\section{References}

1 Kohn R, Saxena S, Levav I, Saraceno B. The treatment gap in mental health care. Bull World Health Organ 2004; 82(11): 858-66.

2 Lora A, Kohn R, Levav I, McBain R, Morris J, Saxena S. Service availability and utilization and treatment gap for schizophrenic disorders: a survey in 50 lowand middle-income countries. Bull World Health Organ 2012; 90(1): 47-54, 54A-B.

3 Andrews G, Henderson S. Unmet Need in Psychiatry: Problems, Resources, Responses. Cambridge University Press, 2000

4 Patel V, Maj M, Flisher AJ, De Silva MJ, Koschorke M, Prince M, et al. Reducing the treatment gap for mental disorders: a WPA survey. World Psychiatry 2010; 9(3): 169-76

5 Saxena S, Maj M. Physical health of people with severe mental disorders: leave no one behind. World Psychiatry 2017; 16(1): 1-2.

6 Correll CU, Solmi M, Veronese N, Bortolato B, Rosson S, Santonastaso P, et al. Prevalence, incidence and mortality from cardiovascular disease in patients with pooled and specific severe mental illness: a large-scale meta-analysis of $3,211,768$ patients and 113,383,368 controls. World Psychiatry 2017; 16(2): $163-80$. 
7 Haklai Z, Goldberger N, Stein N, Pugachova I, Levav I. The mortality risk among persons with psychiatric hospitalizations. Isr J Psychiatry Relat Sci 2011; 48(4): 230-9.

8 DiMatteo MR, Hays RD, Gritz ER, Bastani R, Crane L, Elashoff R, et al. Patient adherence to cancer control regimens: scale development and initial validation. Psychol Assess 1993; 5(1): 102-12.

9 Dolgin MJ, Katz ER, Doctors SR, Siegel SE. Caregivers' perceptions of medical compliance in adolescents with cancer. J Adolesc Health Care 1986; 7(1): 22-7.

10 Jamison RN, Lewis S, Burish TG. Cooperation with treatment in adolescent cancer patients. J Adolesc Health Care 1986; 7(3): 162-7.

11 Glasgow RE, Toobert DJ, Riddle M, Donnelly J, Mitchell DL, Calder D. Diabetesspecific social learning variables and self-care behaviors among persons with type II diabetes. Health Psychol 1989; 8(3): 285-303.

12 Lora A, Monzani E, Ibrahim B, Soranna D, Corrao G. Routine quality care assessment of schizophrenic disorders using information systems. Int J Qual Health Care 2016; 28(6): 728-33

13 Doherty AM, Gaughran F. The interface of physical and mental health. SOC Psychiatry Psychiatr Epidemiol 2014; 49(5): 673-82.

14 Von Korff M, Ormel J, Katon W, Lin EH. Disability and depression among high utilizers of health care. a longitudinal analysis. Arch Gen Psychiatry 1992; 49(2): 91-100.

15 Simonsick EM, Wallace RB, Blazer DG, Berkman LF. Depressive symptomatology and hypertension-associated morbidity and mortality in older adults. Psychosom Med 1995; 57(5): 427-35.

16 Corrao G, Barbato A, D'Avanzo B, Di Fiandra T, Ferrara L, Gaddini A, et al. Does the mental health system provide effective coverage to people with schizophrenic disorder? A self-controlled case series study in Italy. Soc Psychiatry Psychiatr Epidemiol [Epub ahead of print] 16 Jun 2021. Available from: https://doi.org/10.1007/s00127-021-02114-9.

17 DiMatteo MR, Giordani PJ, Lepper HS, Croghan TW. Patient adherence and medical treatment outcomes: a meta-analysis. Med Care 2002; 40(9): 794-811.

18 Newby LK, LaPointe NMA, Chen AY, Kramer JM, Hammill BG, DeLong ER, et al. Long-term adherence to evidence-based secondary prevention therapies in coronary artery disease. Circulation 2006; 113(2): 203-12.

19 Suissa S. Immeasurable time bias in observational studies of drug effects on mortality. Am J Epidemiol 2008; 168(3): 329-35.

20 American Diabetes Association. Standards of medical care in diabetes. Diabetes Care 2017; 40(suppl 1): 1-142.

21 Moffet HH, Parker MM, Sarkar U, Schillinger D, Fernandez A, Adler NE, et al. Adherence to laboratory test requests by patients with diabetes: the Diabetes Study of Northern California (DISTANCE). Am J Manag Care 2011; 17(5): 339-44

22 Corrao G, Rea F, Di Martino M, Lallo A, Davoli M, De Palma R, et al. Effectiveness of adherence to recommended clinical examinations of diabetic patients in preventing diabetes-related hospitalizations. Int J Qual Health Care 2019; 31(6): 464-72.

23 Corrao G, Rea F, Di Martino M, De Palma R, Scondotto S, Fusco D, et al. Developing and validating a novel Multisource Comorbidity Score from administrative data: a large population-based cohort study from Italy. BMJ Open 2017; 7(12): e019503
24 Austin PC. Using the standardized difference to compare the prevalence of a binary variable between two groups in observational research. Commun Stat Simul Comput 2009; 38(6): 1228-34.

25 Wheeler AJ, O'Reilly CL, El-Den S, Byrnes J, Ware RS, McMillan SS. Bridging the gap between physical and mental illness in community pharmacy (PharMlbridge): protocol for an Australian cluster randomised controlled trial. BMJ Open 2020; 10(7): e039983.

26 Wade M, Tai S, Awenat $Y$, Haddock G. A systematic review of service-user reasons for adherence and nonadherence to neuroleptic medication in psychosis. Clin Psychol Rev 2017; 51: 75-95.

27 Ehrlich C, Chester P, Kisely S, Crompton D, Kendall E. Making sense of self-care practices at the intersection of severe mental illness and physical health-an Australian study. Health Soc Care Community 2018; 26(1): e47-55.

28 Morant N, Azam K, Johnson S, Moncrieff J. The least worst option: user experiences of antipsychotic medication and lack of involvement in medication decisions in a UK community sample. J Ment Health 2018; 27(4): 322-8.

29 Brown S, Kim M, Mitchell C, Inskip H. Twenty-five year mortality of a community cohort with schizophrenia. Br J Psychiatry 2010; 196(2): 116-21.

30 Miller C, Bauer MS. Excess mortality in bipolar disorders. Curr Psychiatry Rep 2014; 16(11): 499.

31 McGrath J, Saha S, Chant D, Welham J. Schizophrenia: a concise overview of incidence, prevalence, and mortality. Epidemiol Rev 2008; 30: 67-76.

32 Saha S, Chant D, McGrath J. A systematic review of mortality in schizophrenia: is the differential mortality gap worsening over time? Arch Gen Psychiatry 2007; 64(10): 1123-31.

33 Osby U, Brandt L, Correia N, Ekbom A, Sparén P. Excess mortality in bipolar and unipolar disorder in Sweden. Arch Gen Psychiatry 2001; 58(9): 844-50.

34 Roshanaei-Moghaddam B, Katon W. Premature mortality from general medical illnesses among persons with bipolar disorder: a review. Psychiatr Serv 2009; 60(2): 147-56.

35 Hoang U, Goldacre MJ, Stewart R. Avoidable mortality in people with schizophrenia or bipolar disorder in England. Acta Psychiatr Scand 2013; 127(3): 195-201.

36 Kurowecki D, Godbout J. Dying young: excess morbidity and mortality in individuals with severe mental illness and what we should be doing about it. Univ Ott J Med 2014; 4.

37 Saha S. Avoidable vs. non-avoidable causes of deaths in schizophrenia and bipolar disorders. Acta Psychiatr Scand 2013; 127(3): 171-2.

38 DE Hert M, Correll CU, Bobes J, Cetkovich-Bakmas M, Cohen D, Asai I, et al. Physical illness in patients with severe mental disorders. I. Prevalence, impact of medications and disparities in health care. World Psychiatry 2011; 10(1): 52-77.

39 Nolte E, McKee CM. In amenable mortality-deaths avoidable through health care-progress in the US lags that of three European countries. Health Aff 2012; 31(9): 2114-22.

40 Corrao G, Zambon A, Parodi A, Mezzanzanica M, Merlino L, Cesana G, et al. Do socioeconomic disparities affect accessing and keeping antihypertensive drug therapy? Evidence from an Italian population-based study. J Hum Hypertens 2009; 23(4): 238-44.

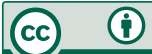

